\title{
Impact of tumor programmed death ligand-1 expression on osimertinib efficacy in untreated EGFR-mutated advanced non-small cell lung cancer: a prospective observational study
}

\author{
Akihiro Yoshimura ${ }^{1}$, Tadaaki Yamada ${ }^{1}$, , Yusuke Okuma ${ }^{2,3}$, Akito Fukuda $^{2,3}$, Satoshi Watanabe ${ }^{4}$ \\ Naoya Nishioka ${ }^{5}$, Takayuki Takeda ${ }^{6}$, Yusuke Chihara $^{7}$, Shinnosuke Takemoto ${ }^{8}$, Taishi Harada ${ }^{9}$, \\ Osamu Hiranuma ${ }^{10}$, Yukina Shirai ${ }^{11}$, Akihiro Nishiyama ${ }^{12}$, Seiji Yano ${ }^{12}$, Yasuhiro Goto ${ }^{13}$, \\ Shinsuke Shiotsu ${ }^{14}$, Kei Kunimasa ${ }^{15}$, Yoshie Morimoto ${ }^{1}$, Masahiro Iwasaku ${ }^{1}$, Yoshiko Kaneko ${ }^{1}$, \\ Junji Uchino ${ }^{1}$, Hirotsugu Kenmotsu ${ }^{5}$, Toshiaki Takahashi ${ }^{5}$, Koichi Takayama ${ }^{1}$
}

${ }^{1}$ Department of Pulmonary Medicine, Graduate School of Medical Science, Kyoto Prefectural University of Medicine, Kyoto, Japan; ${ }^{2}$ Department of Thoracic Oncology and Respiratory Medicine, Tokyo Metropolitan Cancer and Infectious Diseases Center Komagome Hospital, Tokyo, Japan; ${ }^{3}$ Department of Thoracic Oncology, National Cancer Center Hospital, Tokyo, Japan; ${ }^{4}$ Department of Respiratory Medicine and Infectious Diseases, Niigata University Graduate School of Medicine and Dental Hospital, Niigata, Japan; ${ }^{5}$ Division of Thoracic Oncology, Shizuoka Cancer Center, Shizuoka, Japan; ${ }^{6}$ Department of Respiratory Medicine, Japanese Red Cross Kyoto Daini Hospital, Kyoto, Japan; ${ }^{7}$ Department of Respiratory Medicine, Uji-Tokushukai Medical Center, Kyoto, Japan; ${ }^{8}$ Department of Respiratory Medicine, Nagasaki University Graduate School of Biomedical Sciences, Nagasaki, Japan; ${ }^{9}$ Department of Medical Oncology, Fukuchiyama City Hospital, Kyoto, Japan; ${ }^{10}$ Department of Respiratory Medicine, Otsu City Hospital, Shiga, Japan; ${ }^{11}$ Department of Respiratory Medicine, Juntendo University, Tokyo, Japan; ${ }^{12}$ Division of Medical Oncology, Cancer Research Institute, Kanazawa University, Kanazawa, Japan; ${ }^{13}$ Department of Respiratory Medicine, Fujita Health University School of Medicine, Aichi, Japan; ${ }^{14}$ Department of Respiratory Medicine, Japanese Red Cross Kyoto Daiichi Hospital, Kyoto, Japan; ${ }^{15}$ Department of Thoracic Oncology, Osaka International Cancer Institution, Osaka, Japan

Contributions: (I) Conception and design: T Yamada, K Takayama; (II) Administrative support: T Yamada; (III) Provision of study materials or patients: A Yoshimura, Y Okuma, A Fukuda, S Watanabe, N Naoya, T Takeda, Y Chihara, S Takemoto, T Harada, O Hiranuma, Y Shirai, A Nishiyama, S Yano, Y Goto, S Shiotsu, K Kunimasa, H Kenmotsu, T Takahashi; (IV) Collection and assembly of data: A Yoshimura; (V) Data analysis and interpretation: A Yoshimura, T Yamada, Y Okuma, Y Morimoto, M Iwasaku, Y Kaneko, J Uchino, H Kenmotsu, T Takahashi, K Takayama; (VI) Manuscript writing: All authors; (VII) Final approval of manuscript: All authors.

Correspondence to: Tadaaki Yamada, MD, PhD. Department of Pulmonary Medicine, Graduate School of Medical Science, Kyoto Prefectural University of Medicine, 465, Kajii-cho, Kamigyo-ku, Kyoto, 602-8566, Japan. Email: tayamada@koto.kpu-m.ac.jp.

\begin{abstract}
Background: Osimertinib monotherapy is currently the standard of care as a first-line treatment for patients harboring epidermal growth factor receptor (EGFR) mutations; however, some EGFR-mutated non-small cell lung cancer (NSCLC) patients exhibit primary resistance and an insufficient response to EGFR-tyrosine kinase inhibitors (EGFR-TKIs). Elevated programmed death-ligand 1 (PD-L1) expression in tumors was reported as a negative predictive factor for outcomes of first- or second-generation EGFR-TKIs.

Methods: We prospectively assessed advanced NSCLC patients with EGFR mutations who were treated with osimertinib at 14 institutions in Japan between September 2019 and December 2020. Relationships between outcomes of osimertinib monotherapy and patients' characteristics were reviewed.

Results: Seventy-one patients who underwent the tumor PD-L1 test were enrolled. Multivariate analysis identified tumor PD-L1 expression as an independent predictor for progression-free survival (PFS) with osimertinib treatment $(\mathrm{P}=0.029)$. The objective-response and disease-control rates for osimertinib treatment were significantly lower in patients demonstrating elevated PD-L1 levels relative to those with low or negative PD-L1 level $(\mathrm{P}=0.043$ and $\mathrm{P}=0.007$, respectively). Furthermore, among patients treated with
\end{abstract}

^ ORCID: Akihiro Yoshimura, 0000-0002-3753-2110; Tadaaki Yamada, 0000-0002-6945-281X. 
osimertinib, those with high PD-L1 levels exhibited shorter PFS relative to those with low plus negative PDL1 level (median PFS: 5.0 vs. 17.4 months; $\mathrm{P}<0.001$ ).

Conclusions: Elevated tumor PD-L1 expression is associated with poor outcomes of osimertinib monotherapy in previously untreated advanced NSCLC patients with EGFR mutation. Further clinical trials are warranted to accumulate evidence demonstrating the effectiveness of combination therapy with osimertinib for EGFR-mutated advanced NSCLC patients with elevated tumor PD-L1 expression.

Trial Registration: UMIN000043942.

Keywords: EGFR mutation; osimertinib; programmed death ligand-1 (PD-L1); non-small cell lung cancer (NSCLC); biomarker

Submitted Jun 03, 2021. Accepted for publication Aug 11, 2021.

doi: $10.21037 /$ tlcr-21-461

View this article at: https://dx.doi.org/10.21037/tlcr-21-461

\section{Introduction}

Lung cancer is the number one cause of cancer-related death worldwide (1), and non-small cell lung cancer (NSCLC) is the most common subtype, accounting for $\sim 85 \%$ of all lung cancer cases (2). Improved clinical outcomes in NSCLC patients harboring epidermal growth factor receptor (EGFR) mutations, including major subtypes, such as exon 19 deletion or point mutation in exon 21 resulting in L858R substitution, have contributed to the development of EGFR-targeted therapy. NSCLC patients with activating EGFR mutations exhibited better responses to first- and second-generation EGFR-tyrosine kinase inhibitors (EGFR-TKIs) than to systemic platinum-based chemotherapy $(3,4)$. Treatment with the third-generation EGFR-TKI osimertinib showed better outcomes than those with first-generation EGFR-TKIs, such as gefitinib or erlotinib, in first-line treatment of advanced EGFRmutated NSCLC patients (5). Therefore, osimertinib has been approved as a therapy for untreated EGFRmutated advanced NSCLC patients in countries, including the United States and Japan. Although osimertinib monotherapy represents a promising treatment modality, $\sim 20 \%$ of EGFR-mutated NSCLC patients exhibit primary resistance to osimertinib (5). To date, other therapeutic strategies have been approved in several countries, including the USA and Japan, for first-line treatment of EGFRmutated NSCLC patients, including initial combination therapy with an anti-angiogenesis agent and chemotherapy to overcome the above issues and other EGFR-TKIs $(6,7)$. Therefore, it is of important clinical relevance to determine an optimal initial therapeutic strategy for patients with EGFR-mutated advanced NSCLC.
Although EGFR-mutated advanced NSCLC cells respond well to osimertinib initially, a small percentage of cells can survive and expand, leading to acquired drug resistance and tumor heterogeneity, ultimately promoting tumor recurrence. As for intrinsic resistance to EGFRTKIs, EGFR-T790M mutation, EGFR-exon20 insertions, and BIM deletion polymorphism have been reported as contributory factors (8-10). Based on previous reports, acquired-resistance mechanisms can be broadly classified into resistance caused by the treatment target EGFR [EGFR-T790M secondary resistance gene mutation (11)], resistance via non-EGFR bypass signal [Met gene amplification (12), HGF overexpression (13), HER2 gene amplification (14), GAS6-AXL signal activation (15)], and other resistance [transformation to small cell lung cancer (16) and epithelial-to-mesenchymal transition (17)].

Recently, immune-checkpoint inhibitor (ICI) therapy has made rapid advances in several cancers, including lung cancer, according to improved clinical outcomes, such as prolonged survival and a more durable treatment response (18-22). The identification of promissing biomarkers for detecting respondents to ICI treatment is currently underway, with programmed death ligand-1 (PD-L1) expression in tumors clinically identified as a positive predictive biomarker for advanced NSCLC patients treated with ICIs, especially for NSCLC patients with wild-type driver oncogenes (21). Elevated PD-L1 expression in tumors suppresses T cell activation and growth via apoptosis of effector $\mathrm{T}$ cells, which interferes with tumor immune responses $(23,24)$, thereby identifying PD-L1 as a negative regulator of immune response. Preclinical studies have shown that activation of EGFR signaling pathways is involved in the induction of 
PD-L1 expression in NSCLC cells (25). Meanwhile, tumor PD-L1 expression was identified as a negative predictor of outcome in EGFR-mutated advanced NSCLC patients treated with first- or second-generation EGFR-TKIs (26-30). However, the effect of tumor PD-L1 level on the efficacy of osimertinib monotherapy in EGFR-mutated advanced NSCLC patients remains unknown. In this prospective study, we identified biomarkers of osimertinib efficacy as first-line treatment for EGFR-mutated advanced NSCLC patients. We present the following article in accordance with the STROBE reporting checklist (available at https://dx.doi.org/10.21037/tlcr-21-461).

\section{Methods}

\section{Patients}

We prospectively assessed 71 advanced or postoperative recurrent NSCLC patients harboring an EGFRactivating mutation, who were treated with osimertinib at 14 institutions in Japan between September 2019 and December 2020. Osimertinib administration and assessment of its efficacy and toxicity were performed by each investigator. Image evaluation was stipulated by every 8 to 12 weeks, including complete response, partial response, stable disease, and progressive disease, using either conventional computed tomography or magnetic resonance imaging, according to criteria outlined in Response Evaluation Criteria in Solid Tumors (v.1.1). Progressionfree survival (PFS) was defined as the time from initiation of osimertinib treatment to the date of objective disease progression or death, regardless of whether the patient withdrew from osimertinib treatment or received another anticancer therapy prior to progression. Among the 70 EGFR-mutant NSCLC patients showing disease progression within 90 days or during $>90$-day follow-up, seven were identified as exhibiting primary resistance to osimertinib treatment and categorized as "disease progression within 90 days". The inclusion criteria in this study are as follows; (I) patients without any systemic treatment, (II) symptomatic brain metastases are allowed, (III) any Eastern Cooperative Oncology Groups performance status (ECOG PS) is allowed, (IV) EGFR mutations, including L858R point mutation in exon 21 and exon 19 deletions, in addition to the other types of mutation, such as G719X in exon18, S768I in exon 20, L861Q in exon 21, were included. This study was conducted in accordance with the
Declaration of Helsinki (as revised in 2013). This study was approved by the institutional review board in Kyoto Prefectural University of Medicine (ERB-C-1242) and each respective hospital and registered at the University Medical Hospital Information Network (UMIN) Clinical Trials Registry (UMIN000043942). In addition, we had performed opt-out informed consent at each hospital from the trial initiation. Written informed consent was obtained from all participants.

\section{EGFR-mutation analysis}

$E G F R$ mutations were detected using polymerase chain reaction (PCR) of tumor samples by sequencing exons 18 through 21, with the sequencing performed in commercial clinical laboratories (SRL, Inc., Tokyo, Japan; and BML, Inc., Tokyo, Japan). Deletions in exon 19 or a leucine to arginine substitution (L858R) in exon 21 are referred to as common mutations, and the other mutations are referred to as uncommon mutations.

\section{Analysis of PD-L1 expression}

PD-L1 expression in tumors was assessed using pretreatment tumor samples by performing PD-L1 immunohistochemistry (IHC) using a 22C3 pharmDx assay at a commercial clinical laboratory (SRL, Inc., Tokyo, Japan). Tumor PD-L1 expression was given as a percentage in at least 100 viable tumor cells used for complete or partial membrane staining. Pathologists at the commercial vendor interpreted tumor PD-L1 expression according to assay results. Patients were categorized into the following three groups based on PD-L1 tumor-proportion score (TPS): high ( $\geq 50 \%)$, low (1-49\%), and negative $(<1 \%)$.

\section{Statistical analysis}

To analyze PFS, times to events were estimated using the Kaplan-Meier method and compared using the logrank test. Hazard ratios (HRs) for PFS were determined using a univariate Cox proportional hazards model. Cox proportional hazards models evaluating several patient factors were used. To construct the multivariate model, we selected the most relevant factors related to PFS, identified in the results of univariate analysis. All statistical analyses were performed using GraphPad Prism software (v.8.0; GraphPad Software, San Diego, CA, USA). P $<0.05$ was 
Table 1 Patients' characteristics

\begin{tabular}{|c|c|}
\hline Characteristics & $\mathrm{n}=71$ \\
\hline Median age, years (range) & $71.0(35.0-87.0)$ \\
\hline \multicolumn{2}{|l|}{ Age categorization, n (\%) } \\
\hline$<75$ & $45(63.4)$ \\
\hline$\geq 75$ & $26(36.6)$ \\
\hline \multicolumn{2}{|l|}{ Sex, n (\%) } \\
\hline Male & $26(36.6)$ \\
\hline Female & $45(63.4)$ \\
\hline \multicolumn{2}{|l|}{ ECOG PS, n (\%) } \\
\hline 0 & $28(39.4)$ \\
\hline 1 & $30(42.3)$ \\
\hline 2,3 & $13(18.3)$ \\
\hline \multicolumn{2}{|l|}{ Disease stage, $\mathrm{n}(\%)$} \\
\hline III & $2(2.8)$ \\
\hline IV & $60(84.5)$ \\
\hline Postoperative relapse & $9(12.7)$ \\
\hline \multicolumn{2}{|l|}{ Brain metastasis, n (\%) } \\
\hline Positive & $21(29.6)$ \\
\hline Negative & $50(70.4)$ \\
\hline \multicolumn{2}{|l|}{ Histology, n (\%) } \\
\hline Adenocarcinoma & $67(94.4)$ \\
\hline Others & $4(5.6)$ \\
\hline \multicolumn{2}{|l|}{ EGFR mutation, $\mathrm{n}(\%)$} \\
\hline 19del & $32(45.1)$ \\
\hline L858R & $36(50.7)$ \\
\hline G719C & $3(4.2)$ \\
\hline \multicolumn{2}{|l|}{ Smoking status, n (\%) } \\
\hline Current or former & $31(43.7)$ \\
\hline Never & $40(56.3)$ \\
\hline \multicolumn{2}{|l|}{ PD-L1 TPS, n (\%) } \\
\hline$\geq 50 \%$ & $15(21.1)$ \\
\hline $1-49 \%$ & $26(36.6)$ \\
\hline$<1 \%$ & $30(42.3)$ \\
\hline
\end{tabular}

ECOG PS, Eastern Cooperative Oncology Groups Performance Status; EGFR, epidermal growth factor receptor; 19del, exon 19 deletion; L858R, exon 21 L858R mutation; G719C, exon18 G719C mutation; PD-L1, programmed death-ligand 1; TPS, tumor proportion score. considered significant.

\section{Results}

\section{Patient characteristics}

The median age of the $71 E G F R$-mutant advanced NSCLC patients enrolled in this study was 71.0 years (range, 35.0-87.0 years). Forty-five patients (63.4\%) were female. Most patients $(81.7 \%)$ indicated an ECOG PS of 0 or 1 , and 40 patients $(56.3 \%)$ were non-smokers (Table 1). The most prevalent history of disease included incidence of adenocarcinoma (94.4\%), and 9 patients $(12.7 \%)$ experienced relapse after surgery. According to EGFR-mutation status, 32 patients $(45.1 \%)$ harbored exon 19 deletion, 36 patients $(50.7 \%)$ harbored a point mutation in exon 21 resulting in L858R substitution, and 3 patients (4.2\%) had a point mutation in exon 18 at G719C (uncommon).

\section{Predictive factor for initial osimertinib treatment in EGFR-mutant advanced NSCLC patients}

We then examined the predictive factors of osimertinib treatment in EGFR-mutant advanced NSCLC patients. The median follow-up time for this study was 15.5 months (range, 1.2-25.1 months). Fifty-four patients were followed up for more than 1 year, and 3 patients for more than 2 years (Figure S1). Median overall survival time (OS) was not evaluable (NE) (95\% CI: 22.4-NE) (Figure S2A), and 17 patients $(23.9 \%)$ were successively treated. Univariate analysis identified ECOG PS, EGFR-mutation status, and tumor PD-L1 expression as predictors of PFS for osimertinib monotherapy $(\mathrm{P}=0.010, \mathrm{P}<0.001$, and $\mathrm{P}=0.003$, respectively) (Table 2 ), and multivariate analysis demonstrated that EGFR-mutation status and PD-L1 expression were independent predictive factors for PFS in osimertinib treatment [HR: 2.05, 95\% confidence interval (CI): 1.06-3.97, $\mathrm{P}=0.034$; and HR: $2.40,95 \% \mathrm{CI}$ : $1.09-5.25, \mathrm{P}=0.029$, respectively] (Table 3). These findings demonstrated that tumor PD-L1 expression was related to the efficacy of osimertinib treatment in EGFR-mutated NSCLC patients.

\section{The significance of tumor PD-L1 expression on clinicopathological features and osimertinib efficacy}

Of the 71 patients, 15, 26, and 30 patients were classified 
Table 2 Cox proportional hazard models for PFS in patients with non-small cell lung cancer harbording EGFR mutation who received osimertinib monotherapy, univariate analysis

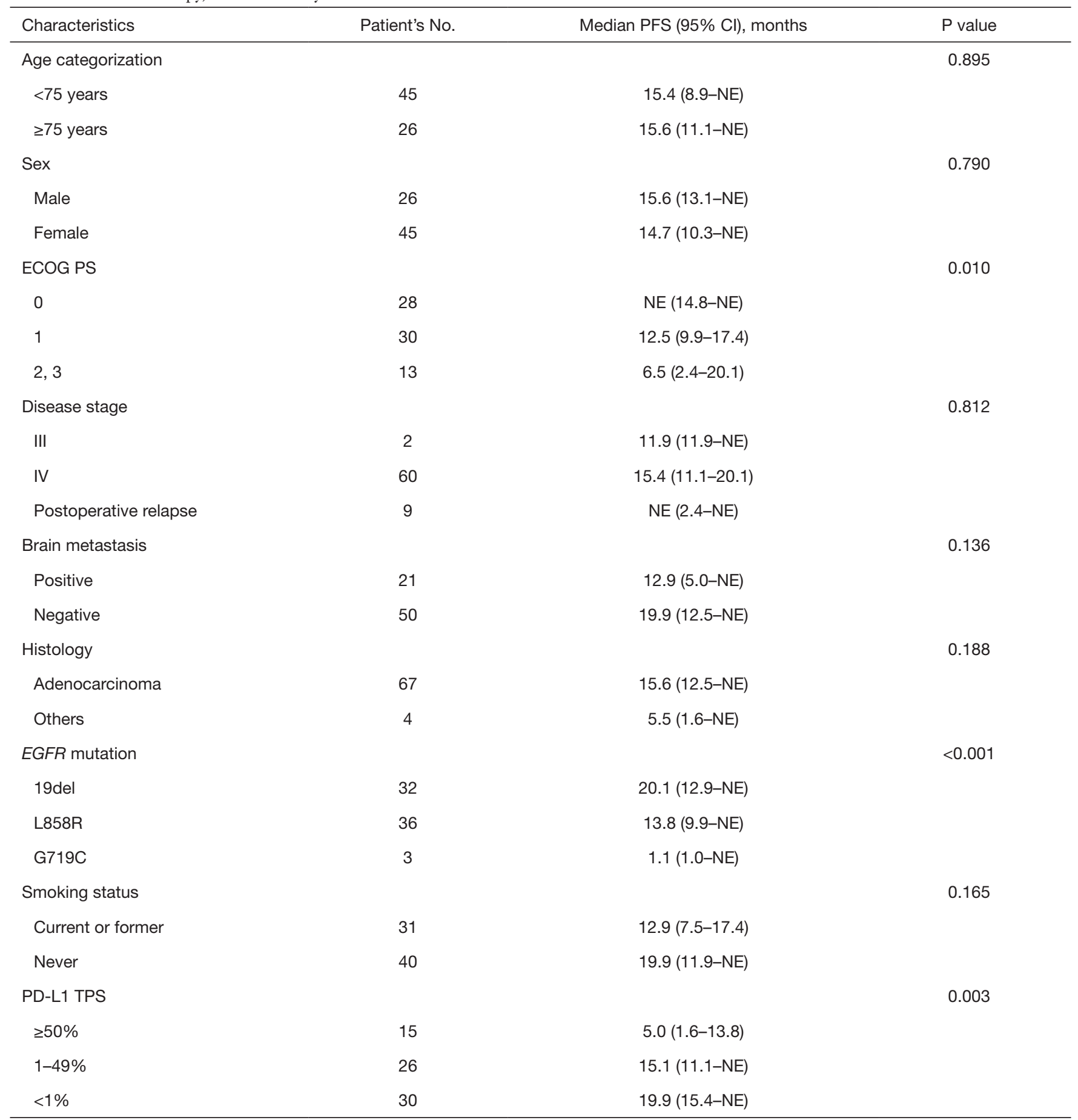

PFS, progression-free survival; EGFR, epidermal growth factor receptor; Cl, confidential interval; NE, not evaluable; ECOG PS, Eastern Cooperative Oncology Groups Performance Status; 19del, exon 19 deletion; L858R, exon 21 L858R mutation; G719C; exon18 G719C mutation, PD-L1, programmed death-ligand 1; TPS, tumor proportion score. 
Table 3 Cox proportional hazard models for PFS in patients with non-small cell lung cancer harbording EGFR mutation who received osimertinib monotherapy, multivariate analysis

\begin{tabular}{lll}
\hline Items & PFS, hazard ratio $(95 \%$ Cl) & P value \\
\hline ECOG PS $\geq 2$ & $1.71(0.78-3.73)$ & 0.180 \\
EGFR mutation status (19del vs. L858R vs. uncommon mutation) & $2.05(1.06-3.97)$ & 0.034 \\
PD-L1 TPS $\geq 50 \%{ }^{\mathrm{a}}$ & $2.40(1.09-5.25)$ & 0.029 \\
\hline
\end{tabular}

a , PD-L1 TPS $\geq 50 \%$ versus all others except for unknown. PFS, progression-free survival; EGFR, epidermal growth factor receptor; Cl, confidence interval; ECOG PS, Eastern Cooperative Oncology Groups Performance Status; 19del, exon 19 deletion; L858R, exon21 L858R mutation; PD-L1, programed death-ligand 1; TPS, tumor proportional score.

into PD-L1 TPS high ( $\geq 50 \%)$, low (1-49\%), and negative $(<1 \%)$ groups, respectively. We assessed correlations of clinicopathological features by comparing the PD-L1 groups. There was no significant difference between the three groups (Table 4).

We then examined the effect of tumor PD-L1 expression on osimertinib efficacy. In all EGFR-mutated NSCLC patients, the objective-response rate (ORR) and diseasecontrol rate (DCR) for osimertinib treatment were $72.1 \%$ and $92.6 \%$, respectively (Table S1). The ORR values for osimertinib treatment tended to be low in high-PD-L1 patients compared to those in PD-L1-low and -negative patients (high, low, and negative: $53.3 \%, 88.0 \%$, and $75.0 \%$, respectively; $\mathrm{P}=0.051$ ). Additionally, the DCR values for osimertinib treatment were significantly lower in high-PD-L1 patients than those in PD-L1-low and negative patients (high, low, and negative: $73.3 \%, 100.0 \%$, and $96.4 \%$, respectively; $\mathrm{P}=0.007$ ) (Figure $1 \mathrm{~A}$ and Table 4). Moreover, the ORR values for osimertinib treatment were significantly lower in high-PD-L1 patients relative to both those in PD-L1-low and -negative patients (53.3\% vs. $81.1 \% ; \mathrm{P}=0.043$ ), and the $\mathrm{DCR}$ values for osimertinib treatment were significantly lower in high-PD-L1 patients relative to those in both PD-L1-low and -negative patients (73.3\% vs. 98.1\%; $\mathrm{P}=0.007$ ) (Figure $1 B$ ).

The frequency of primary resistance to osimertinib treatment was significantly higher in high-PD-L1 patients compared to that in PD-L1-low and -negative patients $(33.33 \%, 3.85 \%$, and $3.45 \%$, respectively; $\mathrm{P}=0.006)$ (Figure 1C).

Median PFS with osimertinib treatment was 15.4 months [95\% CI: 11.9-not evaluable (NE)] in all EGFR-mutated NSCLC patients (Figure S2B). Notably, osimertinib treatment of NSCLC patients with high PD-L1 expression (5.0 months; 95\% CI: 1.6-13.8) resulted in shorter PFS relative to that of PD-L1-low and -negative patients (low: 15.1 months, 95\% CI: 11.1-NE; and negative: 19.9 months, $95 \%$ CI: $15.3-\mathrm{NE}$, respectively) (high $v s$. low and high $v s$. negative; $\mathrm{P}=0.006$ and $\mathrm{P}=0.003$, respectively) (Figure 1D). Additionally, osimertinib treatment of NSCLC patients with high PD-L1 expression resulted in significantly shortened PFS as compared with that of both PD-L1-low and -negative patients $(<50 \% ; 17.4$ months, 95\% CI: 13.1-NE; $\mathrm{P}<0.001$ ) (Figure $1 E$ ). There was no significant relationship in OS between PD-L1-high patients and $\mathrm{PD}-\mathrm{L} 1-\mathrm{low}$ plus negative patients $(\mathrm{P}=0.858)$ (Figure S3).

Median PFS with osimertinib treatment according to EGFR mutational status was 15.4 months (95\% CI: 11.9-NE) in exon 19 deletion and 13.8 months (95\% CI: 9.9-NE) in exon 21 L858R mutation (Figure S4A,S4B). With respect to median PFS according to EGFR-mutation status, we found no significant correlation between PD-L1-high and PDL1-low or -negative patients harboring exon 19 deletion in EGFR ( $\mathrm{P}=0.522)$, whereas median PFS was significantly shorter in PD-L1-high patients relative to that in PD-L1-low and -negative patients $(<50 \%)$ harboring the point mutation in exon 21 (6.5 vs. 15.6 months; $\mathrm{P}=0.024$ ) (Figure S4C,S4D).

\section{Discussion}

The results of this prospective study revealed the clinical impact of elevated tumor PD-L1 expression as a negative predictive factor in determining the clinical outcomes of osimertinib treatment of EGFR-mutant NSCLC patients. To the best of our knowledge, this is the first study reporting that tumor PD-L1 expression is a clinically relevant predictive factor for osimertinib sensitivity.

Preclinical studies show that EGFR-mutant NSCLC cell lines with high PD-L1 expression exhibit induced epithelial-mesenchymal transition and less susceptibility to EGFR-TKIs via activation of transforming growth factor- $\beta$ / 
Table 4 Clinicopathological features comparing tumor PD-L1 expression

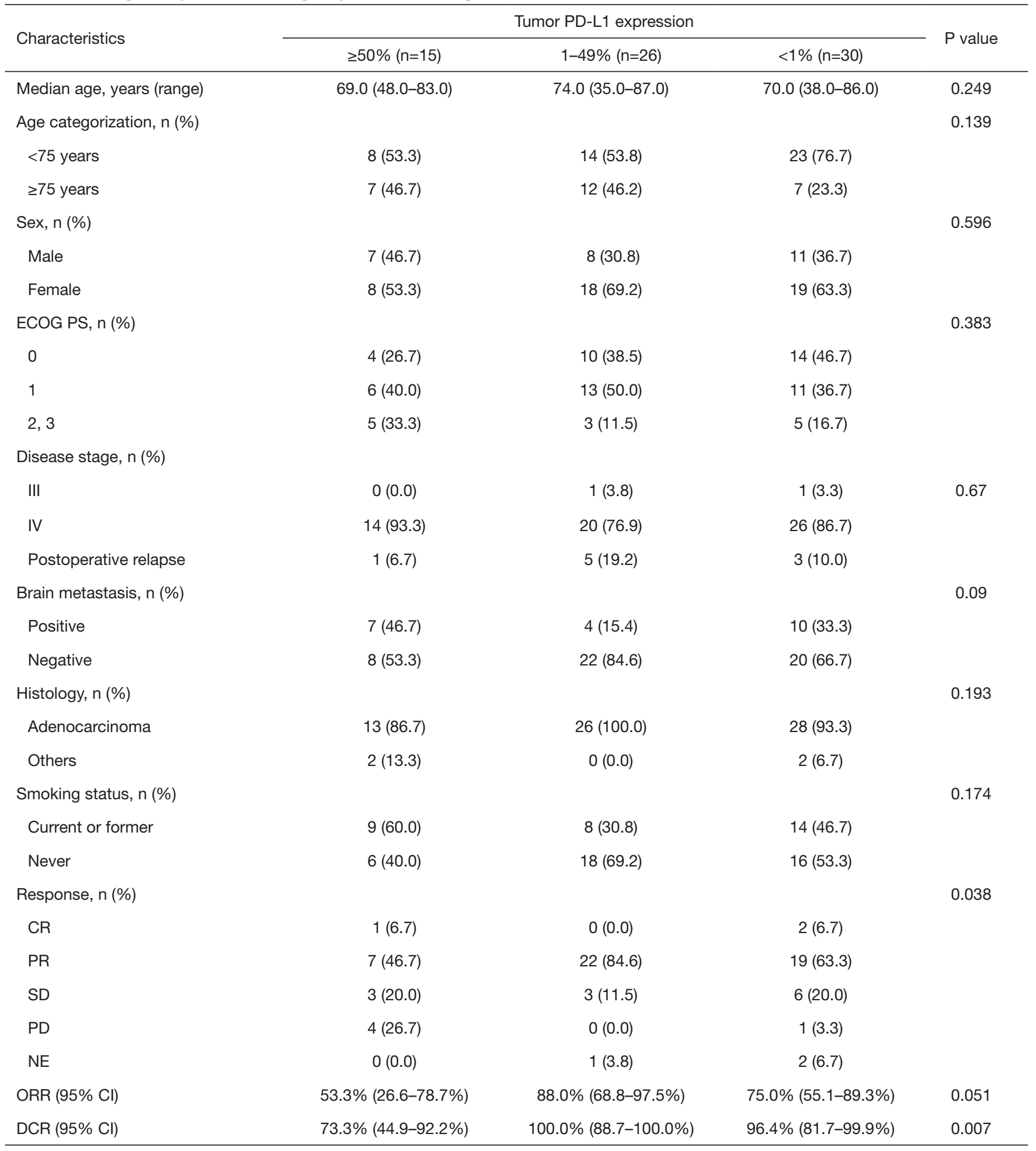

PD-L1, programmed death-ligand 1; ECOG PS, Eastern Cooperative Oncology Groups Performance Status; CR, complete response; PR, partial response; SD, stable disease; PD, progressive disease; NE, not evaluable; ORR, objective response rate; CI, confidence interval; DCR, disease control rate. 

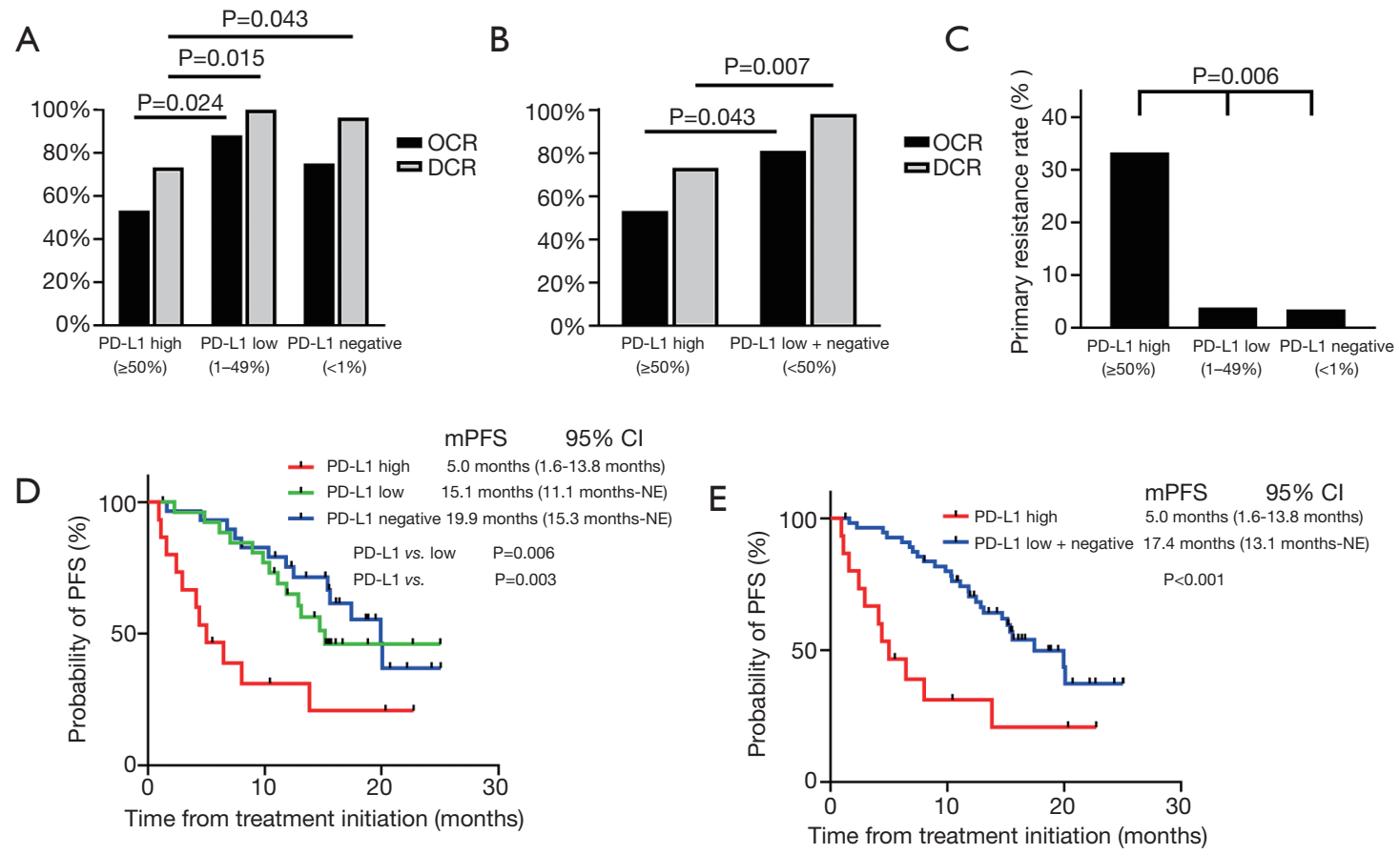

Figure 1 Osimertinib efficacy according to tumor PD-L1 expression. (A) ORR and DCR for osimertinib in PD-L1-high ( $\geq 50 \%$ ), -low (1$49 \%$ ), and -negative ( $<1 \%$ ) patients. (B) ORR and DCR for osimertinib in PD-L1-high and all others group. (C) The frequency of primary resistance to osimertinib treatment in PD-L1-high ( $\geq 50 \%)$, -low (1-49\%), and -negative ( $<1 \%)$ patients. (D) Kaplan-Meier curve for PFS of EGFR-mutated NSCLC patients according to tumor PD-L1 expression (high, low, and negative). Median PFS following osimertinib treatment was 5.0 months (PD-L1-high; 95\% CI: 1.6-13.8), 15.1 months (PD-L1-low; 95\% CI: 11.1-NE), and 19.9 months (PD-L1negative; 95\% CI: 15.3-NE) according to tumor PD-L1 expression (high vs. low and high vs. negative; $\mathrm{P}=0.006$ and $\mathrm{P}=0.003$, respectively). (E) Kaplan-Meier curve for PFS of EGFR-mutated NSCLC patients classified according to tumor PD-L1 expression (high and low + negative). Median PFS following osimertinib treatment was 5.0 months (PD-L1-high; 95\% CI: 1.6-13.8) and 17.4 months (PD-L1-low and -negative; 95\% CI: 13.1-NE) according to tumor PD-L1 expression $(\mathrm{P}<0.001)$. PD-L1, programmed death-ligand 1; ORR, objective response rate; DCR, disease control rate; PFS, progression-free survival; EGFR, epidermal growth factor receptor; NSCLC, non-small cell lung cancer; CI, confidence interval; NE, not evaluable.

SMAD canonical signaling (31). Moreover, previous clinical studies indicated that $E G F R$-mutant NSCLC patients exhibiting $\geq 50 \%$ tumor PD-L1 expression have a shorter PFS following treatment with the first-generation EGFRTKI gefitinib, relative to patients showing tumor PD-L1 expression of $<50 \%$, which agreed with the findings of the present study (26-30). Consistent with these findings, in the present study, we found that high tumor PD-L1 expression $(\geq 50 \%)$ was associated with poor outcomes of EGFRTKI monotherapy in EGFR-mutant NSCLC patients. In contrast, subset analysis of data from the FLAURA clinical trial indicated that the median PFS for EGFR-mutant NSCLC patients with osimertinib was hardly affected between tumor PD-L1 expressors $(\geq 1 \%)$ and negatives $(<1 \%)$ (32). These results suggest that tumor PD-L1 expression of $\geq 50 \%$ might be a potent negative prognostic factor for EGFR-TKI treatment.

Previous studies reported a correlation between EGFRTKI insensitivity and high PD-L1 expression. Specifically, in addition to $E G F R$, activation of other oncogenes promoted EGFR-TKI resistance associated with high PDL1 expression, which led to the accumulation of other genetic alternations. Additionally, evolution of the tumor microenvironment, including immune cells, induced EGFRTKI resistance via loss of tumor-antigen presentation and increased numbers of tumor-associated macrophages as a result of high tumor PD-L1 expression (30,33-35). Moreover, changes in intra-tumoral heterogeneity influence the therapeutic response of EGFR-mutated NSCLC tumors exhibiting high PD-L1 expression to ICIs and EGFR- 
TKIs (36). These observations suggest that the effectiveness of each targeted therapy might be influenced by the resident EGFR mutation or PD-L1 expression of each respective tumor.

To further improve clinical outcomes for EGFR-mutant NSCLC patients, several novel therapeutic approaches are being considered. Elevated tumor PD-L1 expression is a well-known biomarker associated with the response to ICIs, whereas ICI treatment is generally less effective in EGFR-mutated NSCLC patients (37). A previous report showed that tumor PD-L1 expression increases in EGFRmutated NSCLC patients exhibiting high tumor PDL1 expression after attaining resistance to EGFR-TKIs $(38,39)$, suggesting that ICI treatment might be effective in osimertinib-resistant EGFR-mutated NSCLC patients exhibiting high tumor PD-L1 expression. However, another retrospective study showed that the duration of response to previous EGFR-TKIs was a negative predictor of ICI efficacy in EGFR-mutant NSCLC patients (40). Therefore, the utility of PD-L1 expression as a surrogate marker for response to therapeutic PD-L1-blockade in EGFRmutated NSCLC patients remains controversial. Further clinical studies are needed to confirm the response to ICI or combined ICI+osimertinib treatment of EGFR-mutated NSCLC patients, especially those with high tumor PD-L1 expression.

Regulatory T cells (Tregs) are crucial mediators of immune suppression, contribute to tumor immune evasion, and represent poor prognostic factors for various malignancies $(41,42)$. By contrast, treatment with an antivascular endothelial growth factor (VEGF) antibody inhibits Treg proliferation and leads to immune activation, which inactivates Tregs (43). A recent study showed that Treg frequency in tumor microenvironments is a reliable biomarker of clinical responses to the anti-VEGF receptor (VEGFR)2 antibody ramucirumab (44). In a subset analysis of phase 3 trial, the combination of immunochemotherapy plus anti-VEGF antibody bevacizumab improved PFS, compared to immunochemotherapy in advanced NSCLC patients with EGFR mutation [NE (95\% CI: 17.0-NE) vs. 21.4 months (95\% CI: 13.8-NE)] (45). Additionally, several clinical trials demonstrated that the frequency of primary resistance to combination therapy using an antiVEGF/VEGFR antibody and EGFR-TKIs was lower relative to that observed for treatment with EGFR-TKI alone in EGFR-mutated NSCLC patients, suggesting that inhibition of VEGF-related signaling might play an important role in regulating immunomodulatory and/or anti-angiogenic factors $(6,45,46)$. Another study reported that PD-L1 expression is associated with FOXP3expressing Treg infiltration in tumors and poor prognosis in soft tissue sarcoma (47). Therefore, combined therapy with osimertinib and an anti-VEGF/VEGFR antibody might represent a promising therapeutic option for untreated $E G F R$-mutated NSCLC patients exhibiting high tumor PD-L1 expression.

This study has several limitations. First, the study involved a limited cohort of 71 cases, although this is prospective study. Second, all patients in the cohort were Japanese. Third, EGFR mutation status was detected using PCR analysis, which has limitations in the detection of compound mutations. Finally, two patients with a followup time of less than six months were enrolled. However, the novel findings regarding patient response to osimertinib are notable and could be useful for addressing clinical issues.

\section{Conclusions}

Our prospective data demonstrated that tumor PD-L1 expression is significantly associated with osimertinib efficacy in untreated advanced NSCLC patients harboring EGFR mutation. Further clinical trials are required to accumulate clinical evidence demonstrating the effectiveness of combination therapy with osimertinib to improve clinical outcomes for EGFR-mutated advanced NSCLC patients exhibiting high tumor PD-L1 expression.

\section{Acknowledgments}

We would like to thank Editage (www.editage.com) for English language editing.

Funding: None.

\section{Footnote}

Reporting Checklist: The authors have completed the STROBE reporting checklist. Available at https://dx.doi. org/10.21037/tlcr-21-461

Data Sharing Statement: Available at https://dx.doi. org/10.21037/tlcr-21-461

Peer Review File: Available at https://dx.doi.org/10.21037/ tlcr-21-461

Conflicts of Interest: All authors have completed the ICMJE 
uniform disclosure form (available at https://dx.doi. org/10.21037/tlcr-21-461). TY received grants from Pfizer, Ono Pharmaceutical, Chugai Pharmaceutical, and Takeda Pharmaceutical. Satoshi Watanabe received grants and personal fees from AstraZeneca, and Boehringer Ingelheim, personal fees from Chugai Pharmaceutical, Ono Pharmaceutical, Bristol-Myers, Eli Lilly, MSD, Taiho Pharmaceutical, Pfizer, Novartis, and Daiichi Sankyo. Junji Uchino received grants from Eli Lilly, AstraZeneca, and Boehringer Ingelheim. HK received grants and personal fees from Chugai Pharmaceutical, Novartis Pharma, Daiichi-Sankyo, and AstraZeneca, personal fees from Ono Pharmaceutical, Boehringer Ingelheim, Eli Lilly, Kyowa Hakko Kirin, Bristol-Myers, MSD, Pfizer, Taiho Pharma. Toshiaki Takahashi received grants and personal fees from AstraZeneca, Pfizer, Eli Lilly, Chugai Pharmaceutical, Ono Pharmaceutical, MSD, Boehringer Ingelheim, and personal fees from Roche. KT received grants from Chugai Pharmaceutical, and Ono Pharmaceutical, personal fees from AstraZeneca, Chugai Pharmaceutical, MSD, Eli Lilly, Boehringer Ingelheim, and Daiichi Sankyo. The other authors have no conflicts of interest to declare.

Ethical Statement: The authors are accountable for all aspects of the work in ensuring that questions related to the accuracy or integrity of any part of the work are appropriately investigated and resolved. This study was conducted in accordance with the Declaration of Helsinki (as revised in 2013). This study was approved by the institutional review board in Kyoto Prefectural University of Medicine (ERB-C-1242) and each respective hospital and registered at the University Medical Hospital Information Network (UMIN) Clinical Trials Registry (UMIN000043942). In addition, we had performed optout informed consent in each hospital from the beginning of the trial. Written informed consent was obtained from all participation.

Open Access Statement: This is an Open Access article distributed in accordance with the Creative Commons Attribution-NonCommercial-NoDerivs 4.0 International License (CC BY-NC-ND 4.0), which permits the noncommercial replication and distribution of the article with the strict proviso that no changes or edits are made and the original work is properly cited (including links to both the formal publication through the relevant DOI and the license). See: https://creativecommons.org/licenses/by-nc-nd/4.0/.

\section{References}

1. Siegel RL, Miller KD, Jemal A. Cancer statistics, 2020. CA Cancer J Clin 2020;70:7-30.

2. Govindan R, Page N, Morgensztern D, et al. Changing epidemiology of small-cell lung cancer in the United States over the last 30 years: analysis of the surveillance, epidemiologic, and end results database. J Clin Oncol 2006;24:4539-44.

3. Maemondo M, Inoue A, Kobayashi K, et al. Gefitinib or chemotherapy for non-small-cell lung cancer with mutated EGFR. N Engl J Med 2010;362:2380-8.

4. Sequist LV, Yang JC, Yamamoto N, et al. Phase III study of afatinib or cisplatin plus pemetrexed in patients with metastatic lung adenocarcinoma with EGFR mutations. J Clin Oncol 2013;31:3327-34.

5. Soria JC, Ohe Y, Vansteenkiste J, et al. Osimertinib in Untreated EGFR-Mutated Advanced Non-Small-Cell Lung Cancer. N Engl J Med 2018;378:113-25.

6. Nakagawa K, Garon EB, Seto T, et al. Ramucirumab plus erlotinib in patients with untreated, EGFRmutated, advanced non-small-cell lung cancer (RELAY): a randomised, double-blind, placebo-controlled, phase 3 trial. Lancet Oncol 2019;20:1655-69.

7. Hosomi Y, Morita S, Sugawara S, et al. Gefitinib Alone Versus Gefitinib Plus Chemotherapy for Non-Small-Cell Lung Cancer With Mutated Epidermal Growth Factor Receptor: NEJ009 Study. J Clin Oncol 2020;38:115-23.

8. Inukai $\mathrm{M}$, Toyooka $\mathrm{S}$, Ito $\mathrm{S}$, et al. Presence of epidermal growth factor receptor gene T790M mutation as a minor clone in non-small cell lung cancer. Cancer Res 2006;66:7854-8.

9. Yasuda H, Kobayashi S, Costa DB. EGFR exon 20 insertion mutations in non-small-cell lung cancer: preclinical data and clinical implications. Lancet Oncol 2012;13:e23-31.

10. Ng KP, Hillmer AM, Chuah CT, et al. A common BIM deletion polymorphism mediates intrinsic resistance and inferior responses to tyrosine kinase inhibitors in cancer. Nat Med 2012;18:521-8.

11. Kobayashi S, Boggon TJ, Dayaram T, et al. EGFR mutation and resistance of non-small-cell lung cancer to gefitinib. N Engl J Med 2005;352:786-92.

12. Engelman JA, Zejnullahu K, Mitsudomi T, et al. MET amplification leads to gefitinib resistance in lung cancer by activating ERBB3 signaling. Science 2007;316:1039-43.

13. Yano S, Yamada T, Takeuchi S, et al. Hepatocyte 
growth factor expression in EGFR mutant lung cancer with intrinsic and acquired resistance to tyrosine kinase inhibitors in a Japanese cohort. J Thorac Oncol 2011;6:2011-7.

14. Takezawa K, Pirazzoli V, Arcila ME, et al. HER2 amplification: a potential mechanism of acquired resistance to EGFR inhibition in EGFR-mutant lung cancers that lack the second-site EGFRT790M mutation. Cancer Discov 2012;2:922-33.

15. Zhang Z, Lee JC, Lin L, et al. Activation of the AXL kinase causes resistance to EGFR-targeted therapy in lung cancer. Nat Genet 2012;44:852-60.

16. Sequist LV, Waltman BA, Dias-Santagata D, et al. Genotypic and histological evolution of lung cancers acquiring resistance to EGFR inhibitors. Sci Transl Med 2011;3:75ra26.

17. Uramoto H, Iwata T, Onitsuka T, et al. Epithelialmesenchymal transition in EGFR-TKI acquired resistant lung adenocarcinoma. Anticancer Res 2010;30:2513-7.

18. Borghaei H, Paz-Ares L, Horn L, et al. Nivolumab versus Docetaxel in Advanced Nonsquamous Non-Small-Cell Lung Cancer. N Engl J Med 2015;373:1627-39.

19. Brahmer J, Reckamp KL, Baas P, et al. Nivolumab versus Docetaxel in Advanced Squamous-Cell Non-Small-Cell Lung Cancer. N Engl J Med 2015;373:123-35.

20. Herbst RS, Baas P, Kim DW, et al. Pembrolizumab versus docetaxel for previously treated, PD-L1-positive, advanced non-small-cell lung cancer (KEYNOTE-010): a randomised controlled trial. Lancet 2016;387:1540-50.

21. Reck M, Rodríguez-Abreu D, Robinson AG, et al. Pembrolizumab versus Chemotherapy for PD-L1Positive Non-Small-Cell Lung Cancer. N Engl J Med 2016;375:1823-33.

22. Rittmeyer A, Barlesi F, Waterkamp D, et al. Atezolizumab versus docetaxel in patients with previously treated non-small-cell lung cancer (OAK): a phase 3, openlabel, multicentre randomised controlled trial. Lancet 2017;389:255-65.

23. Hatam LJ, Devoti JA, Rosenthal DW, et al. Immune suppression in premalignant respiratory papillomas: enriched functional CD4+Foxp3 + regulatory T cells and PD-1/PD-L1/L2 expression. Clin Cancer Res 2012;18:1925-35.

24. Wenjin Z, Chuanhui P, Yunle W, et al. Longitudinal fluctuations in PD1 and PD-L1 expression in association with changes in anti-viral immune response in chronic hepatitis B. BMC Gastroenterol 2012;12:109.

25. Chen N, Fang W, Zhan J, et al. Upregulation of PD-
L1 by EGFR Activation Mediates the Immune Escape in EGFR-Driven NSCLC: Implication for Optional Immune Targeted Therapy for NSCLC Patients with EGFR Mutation. J Thorac Oncol 2015;10:910-23.

26. Yoneshima Y, Ijichi K, Anai S, et al. PD-L1 expression in lung adenocarcinoma harboring EGFR mutations or ALK rearrangements. Lung Cancer 2018;118:36-40.

27. Su S, Dong ZY, Xie Z, et al. Strong Programmed Death Ligand 1 Expression Predicts Poor Response and De Novo Resistance to EGFR Tyrosine Kinase Inhibitors Among NSCLC Patients With EGFR Mutation. J Thorac Oncol 2018;13:1668-75.

28. Hsu KH, Huang YH, Tseng JS, et al. High PD-L1 expression correlates with primary resistance to EGFRTKIs in treatment naïve advanced EGFR-mutant lung adenocarcinoma patients. Lung Cancer 2019;127:37-43.

29. Liu J, Itchins M, Nagrial A, et al. Relationship between PD-L1 expression and outcome in EGFR-mutant lung cancer patients treated with EGFR tyrosine kinase inhibitors. Lung Cancer 2021;155:28-33.

30. Yang CY, Liao WY, Ho CC, et al. Association between programmed death-ligand 1 expression, immune microenvironments, and clinical outcomes in epidermal growth factor receptor mutant lung adenocarcinoma patients treated with tyrosine kinase inhibitors. Eur J Cancer 2020;124:110-22.

31. Zhang Y, Zeng Y, Liu T, et al. The canonical TGF- $\beta$ / Smad signalling pathway is involved in PD-L1-induced primary resistance to EGFR-TKIs in EGFR-mutant nonsmall-cell lung cancer. Respir Res 2019;20:164.

32. Brown H, Vansteenkiste J, Nakagawa K, et al. Programmed Cell Death Ligand 1 Expression in Untreated EGFR Mutated Advanced NSCLC and Response to Osimertinib Versus Comparator in FLAURA. J Thorac Oncol 2020;15:138-43.

33. Ikeda S, Okamoto T, Okano S, et al. PD-L1 Is Upregulated by Simultaneous Amplification of the PDL1 and JAK2 Genes in Non-Small Cell Lung Cancer. J Thorac Oncol 2016;11:62-71.

34. Frederick DT, Piris A, Cogdill AP, et al. BRAF inhibition is associated with enhanced melanoma antigen expression and a more favorable tumor microenvironment in patients with metastatic melanoma. Clin Cancer Res 2013;19:1225-31.

35. Smith MP, Sanchez-Laorden B, O'Brien K, et al. The immune microenvironment confers resistance to MAPK pathway inhibitors through macrophage-derived TNF $\alpha$. Cancer Discov 2014;4:1214-29. 
36. Kunimasa K, Nakamura H, Sakai K, et al. Heterogeneity of EGFR-mutant clones and PD-L1 highly expressing clones affects treatment efficacy of EGFR-TKI and PD-1 inhibitor. Ann Oncol 2018;29:2145-7.

37. Lee CK, Man J, Lord S, et al. Checkpoint Inhibitors in Metastatic EGFR-Mutated Non-Small Cell Lung Cancer-A Meta-Analysis. J Thorac Oncol 2017;12:403-7.

38. Omori S, Kenmotsu H, Abe M, et al. Changes in programmed death ligand 1 expression in non-small cell lung cancer patients who received anticancer treatments. Int J Clin Oncol 2018;23:1052-9.

39. Han JJ, Kim DW, Koh J, et al. Change in PD-L1 Expression After Acquiring Resistance to Gefitinib in EGFR-Mutant Non-Small-Cell Lung Cancer. Clin Lung Cancer 2016;17:263-270.e2.

40. Ichihara E, Harada D, Inoue K, et al. Characteristics of patients with EGFR-mutant non-small-cell lung cancer who benefited from immune checkpoint inhibitors. Cancer Immunol Immunother 2021;70:101-6.

41. Jang TJ. Progressive Increase of Regulatory T Cells and Decrease of CD8+ T Cells and CD8+ T Cells/Regulatory T Cells Ratio during Colorectal Cancer Development. Korean J Pathol 2013;47:443-51.

42. Pan XD, Mao YQ, Zhu LJ, et al. Changes of regulatory $\mathrm{T}$ cells and FoxP3 gene expression in the aging process and its relationship with lung tumors in humans and mice.
Chin Med J (Engl) 2012;125:2004-11.

43. Terme M, Pernot S, Marcheteau E, et al. VEGFAVEGFR pathway blockade inhibits tumor-induced regulatory T-cell proliferation in colorectal cancer. Cancer Res 2013;73:539-49.

44. Tada Y, Togashi Y, Kotani D, et al. Targeting VEGFR2 with Ramucirumab strongly impacts effector/ activated regulatory $\mathrm{T}$ cells and CD8+ $\mathrm{T}$ cells in the tumor microenvironment. J Immunother Cancer 2018;6:106.

45. Reck M, Mok TSK, Nishio M, et al. Atezolizumab plus bevacizumab and chemotherapy in non-small-cell lung cancer (IMpower150): key subgroup analyses of patients with EGFR mutations or baseline liver metastases in a randomised, open-label phase 3 trial. Lancet Respir Med 2019;7:387-401.

46. Saito H, Fukuhara T, Furuya N, et al. Erlotinib plus bevacizumab versus erlotinib alone in patients with EGFR-positive advanced non-squamous non-small-cell lung cancer (NEJ026): interim analysis of an open-label, randomised, multicentre, phase 3 trial. Lancet Oncol 2019;20:625-35.

47. Que Y, Xiao W, Guan YX, et al. PD-L1 Expression Is Associated with FOXP3+ Regulatory T-Cell Infiltration of Soft Tissue Sarcoma and Poor Patient Prognosis. J Cancer 2017;8:2018-25.
Cite this article as: Yoshimura A, Yamada T, Okuma Y, Fukuda A, Watanabe S, Nishioka N, Takeda T, Chihara Y, Takemoto S, Harada T, Hiranuma O, Shirai Y, Nishiyama A, Yano S, Goto Y, Shiotsu S, Kunimasa K, Morimoto Y, Iwasaku M, Kaneko Y, Uchino J, Kenmotsu H, Takahashi T, Takayama K. Impact of tumor programmed death ligand-1 expression on osimertinib efficacy in untreated EGFR-mutated advanced non-small cell lung cancer: a prospective observational study. Transl Lung Cancer Res 2021;10(8):3582-3593. doi: 10.21037/tlcr-21-461 


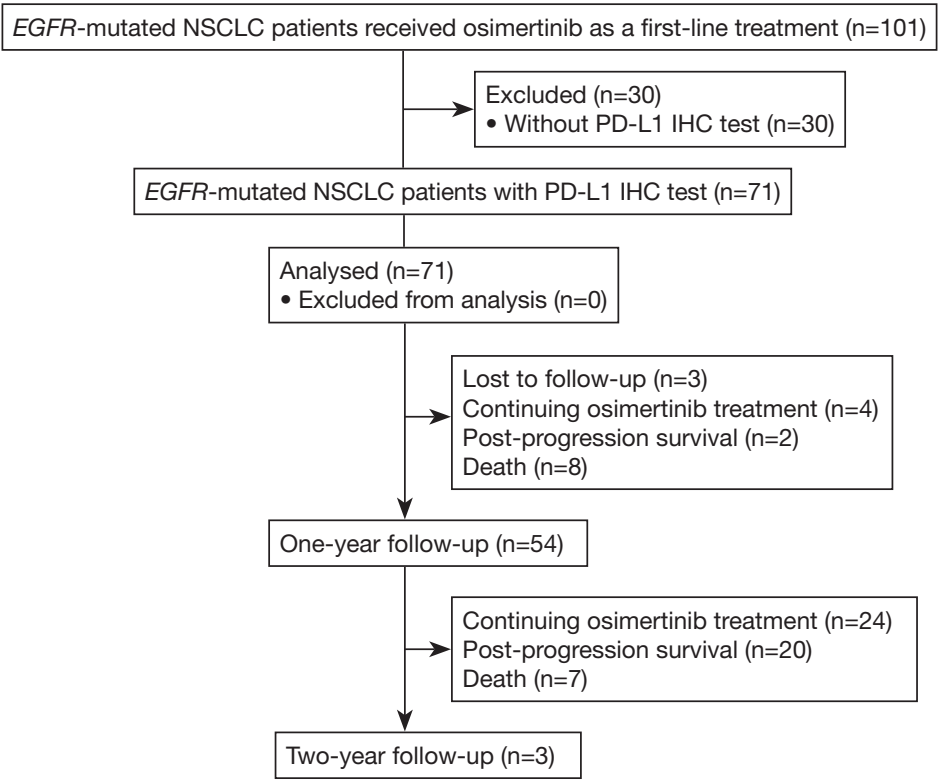

Figure S1 Flow diagram on this prospective study.
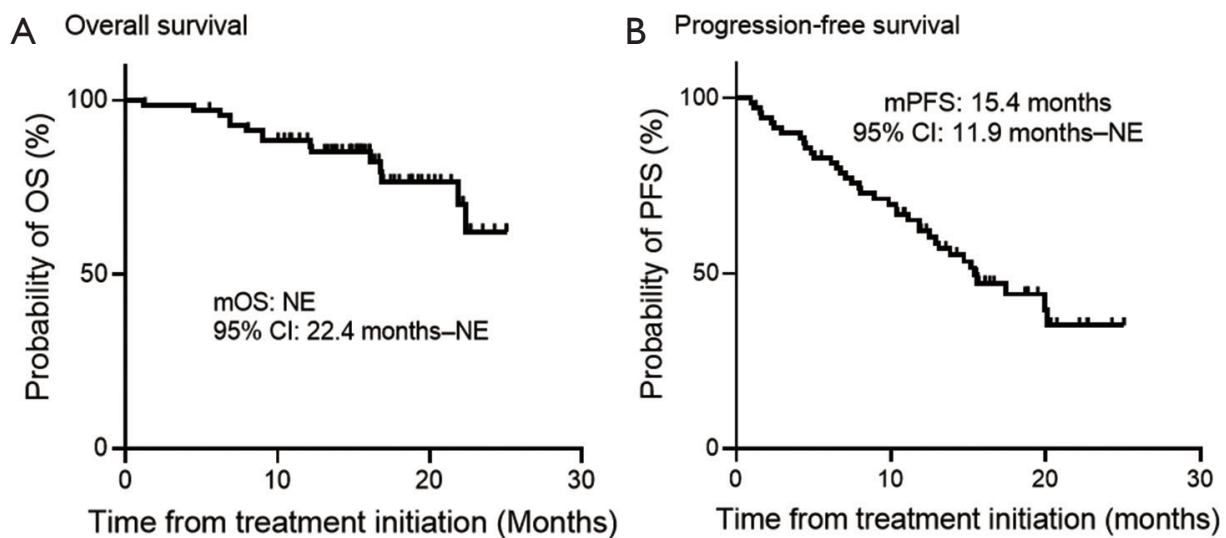

Figure S2 Kaplan-Meier curves for OS and PFS of EGFR mutated non-small cell lung cancer patients who received osimertinib monotherapy. (A) OS in all patients ( $\mathrm{n}=71)$; (B) PFS in all patients ( $\mathrm{n}=71)$. OS, overall survival; PFS, progression-free survival; EGFR, epidermal growth factor receptor; $\mathrm{CI}$, confidence interval; $\mathrm{NE}$, not evaluable. 


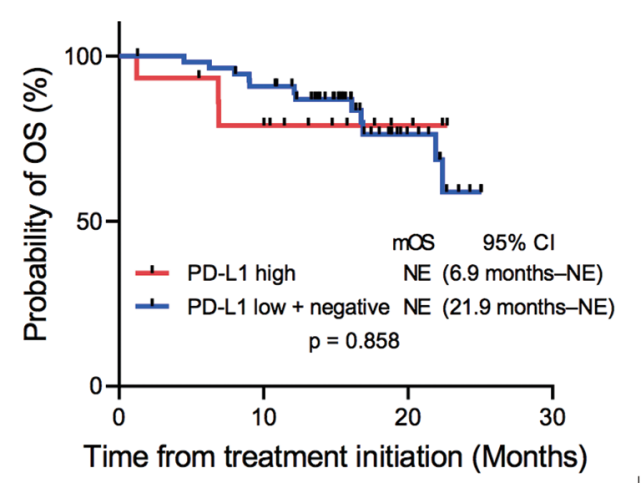

Figure S3 Kaplan-Meier curves for OS of EGFR-mutated non-small cell lung cancer patients who received osimertinib monotherapy between tumor PD-L1 high group and tumor PDL1 low plus negative group. OS, overall survival; EGFR, epidermal growth factor receptor; PD-L1, programmed death-ligand 1; CI, confidence interval; NE, not evaluable.
Table S1 Response to osimertinib for patients with untreated advanced EGFR-mutated non-small cell lung cancer

\begin{tabular}{lc}
\hline Characteristics & $\mathrm{n}=71$ \\
\hline $\mathrm{CR}$ & $2(2.8)$ \\
$\mathrm{PR}$ & $47(66.2)$ \\
$\mathrm{SD}$ & $14(19.7)$ \\
$\mathrm{PD}$ & $5(7.0)$ \\
$\mathrm{NE}$ & $3(4.2)$ \\
ORR $(95 \% \mathrm{Cl})$ & $72.1 \%(59.9-82.3 \%)$ \\
DCR $(95 \% \mathrm{Cl})$ & $92.6 \%(83.7-97.6 \%)$ \\
\hline
\end{tabular}

CR, complete response; PR, partial response; SD, stable disease; PD, progressive disease; NE, not evaluable; ORR, objective response rate; $\mathrm{Cl}$, confidence interval; DCR, disease control rate.
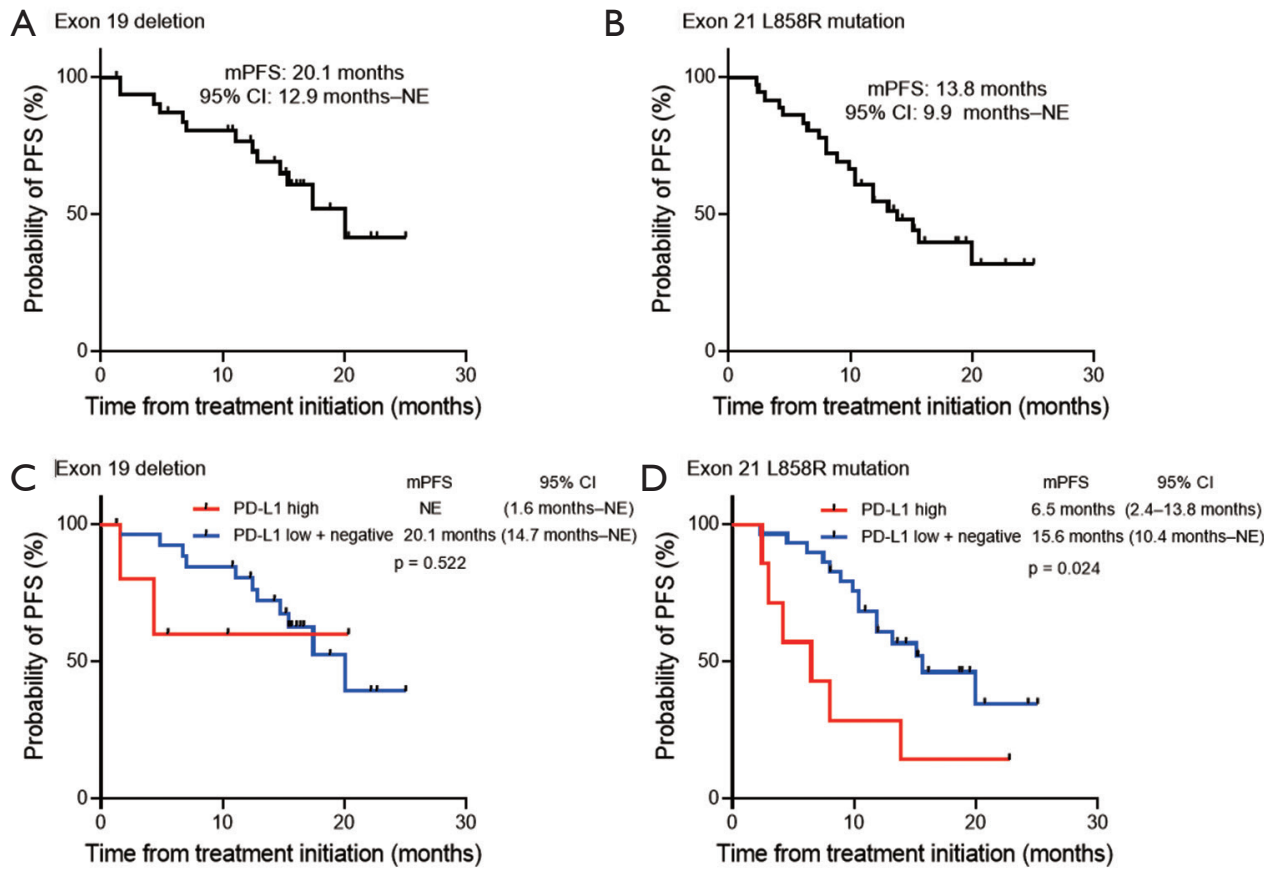

Figure S4 Kaplan-Meier curves for PFS of EGFR-mutated non-small cell lung cancer patients who received osimertinib monotherapy. (A) PFS in patients harboring exon 19 deletion ( $\mathrm{n}=32)$; (B) PFS in patients harboring exon 21 L858R mutation $(\mathrm{n}=36)$; (C) comparison of PFS between tumor PD-L1 high group and tumor PD-L1 low plus negative group in patients harboring exon 19 deletion; (D) comparison of PFS between tumor PD-L1 high group and tumor PD-L1 low plus negative group in patients harboring exon 21 L858R mutation. PFS, progression-free survival; EGFR, epidermal growth factor receptor; PD-L1, programmed death-ligand 1; CI, confidence interval; NE, not evaluable. 